ANNALES

POLONICI MATHEMATICI

$99.3(2010)$

\title{
Infinitely many solutions for a mixed boundary value problem
}

\author{
by Gabriele Bonanno (Messina) \\ and Elisabetta Tornatore (Palermo)
}

\begin{abstract}
The existence of infinitely many solutions for a mixed boundary value problem is established. The approach is based on variational methods.

1. Introduction. Mixed boundary value problems, as well as Dirichlet or Neumann problems, have been widely studied because of their applications to various fields of applied sciences, as mechanical engineering, control systems, computer science, economics, artificial or biological neural networks and many others.

In this connection, several existence and multiplicity results for solutions to second order ordinary differential nonlinear equations, with mixed conditions at the ends, have been investigated making use of fixed point theorems, lower and upper solutions and variational methods (see, for instance, [1], 2], [4], [5], 7], [8]).

The aim of this paper is to establish the existence of infinitely many solutions for mixed boundary value problems by using a very recent critical points theorem (see Theorem 2.1). Our main result (Theorem 3.1), under an appropriate oscillating behaviour of the nonlinear term, ensures an unbounded sequence of solutions for this type of problem. As an example, we present here its special case.
\end{abstract}

TheOREM 1.1. Let $g: \mathbb{R} \rightarrow \mathbb{R}$ be a nonnegative continuous function, put $G(\xi)=\int_{0}^{\xi} g(t) d t$ for all $\xi \in \mathbb{R}$ and assume

$$
\liminf _{\xi \rightarrow+\infty} \frac{G(\xi)}{\xi^{2}}=0, \quad \limsup _{\xi \rightarrow+\infty} \frac{G(\xi)}{\xi^{2}}=+\infty
$$

2010 Mathematics Subject Classification: Primary 34B15.

Key words and phrases: critical points, mixed boundary value problems, infinitely many solutions. 
Then the problem

$$
\left\{\begin{array}{l}
\left.-u^{\prime \prime}+u^{\prime}+u=g(u) \text { in }\right] 0,1[ \\
u(0)=u^{\prime}(1)=0
\end{array}\right.
$$

has a sequence of pairwise distinct classical solutions.

The note is arranged as follows. In Section 2, we recall some basic definitions and our abstract framework, while Section 3 is devoted to the existence of infinitely many solutions for mixed boundary value problems.

2. Preliminaries. Our main tool to investigate the existence of infinitely many solutions for mixed boundary value problems is the infinitely many critical points theorem due to B. Ricceri ([6, Theorem 2.5]). Here, we recall it as given in 3 .

Theorem 2.1 (see [6, Theorem 2.5] and [3, Theorem 2.1]). Let $X$ be a reflexive Banach space, $\Phi: X \rightarrow \mathbb{R}$ be a continuously Gâteaux differentiable, coercive and sequentially weakly lower semicontinuous functional, $\Psi: X \rightarrow \mathbb{R}$ be a sequentially weakly upper semicontinuous and continuously Gâteaux differentiable functional, and $\lambda$ be a positive real parameter.

Put, for each $r>\inf _{X} \Phi$,

$$
\begin{aligned}
\varphi(r) & :=\inf _{u \in \Phi^{-1}(]-\infty, r[)} \frac{\sup _{v \in \Phi^{-1}(]-\infty, r[)} \Psi(v)-\Psi(u)}{r-\Phi(u)}, \\
\gamma & :=\liminf _{r \rightarrow+\infty} \varphi(r), \quad \delta:=\liminf _{\left.r \rightarrow \inf _{X} \Phi\right)^{+}} \varphi(r) .
\end{aligned}
$$

One has

(a) For every $r>\inf _{X} \Phi$ and every $\left.\lambda \in\right] 0,1 / \varphi(r)[$, the restriction of the functional $\Phi-\lambda \Psi$ to $\Phi^{-1}(]-\infty, r[)$ admits a global minimum, which is a critical point (local minimum) of $\Phi-\lambda \Psi$ in $X$.

(b) If $\gamma<\infty$ then, for each $\lambda \in] 0,1 / \gamma[$, the following alternative holds: either

( $\left.\mathrm{b}_{1}\right) \Phi-\lambda \Psi$ possesses a global minimum, or

$\left(\mathrm{b}_{2}\right)$ there is a sequence $\left\{u_{n}\right\}$ of critical points (local minima) of $\Phi-\lambda \Psi$ such that $\lim _{n \rightarrow+\infty} \Phi\left(u_{n}\right)=+\infty$.

(c) If $\delta<\infty$ then, for each $\lambda \in] 0,1 / \delta[$, the following alternative holds: either

$\left(\mathrm{c}_{1}\right)$ there is a global minimum of $\Psi$ which is a local minimum of $\Phi-\lambda \Psi$, or

$\left(c_{2}\right)$ there is a sequence $\left\{u_{n}\right\}$ of pairwise distinct critical points (local minima) of $\Phi-\lambda \Psi$, with $\lim _{n \rightarrow+\infty} \Phi\left(u_{n}\right)=\inf _{X} \Phi$, which weakly converges to a global minimum of $\Phi$. 
Now, consider the following mixed boundary value problem:

$$
\left\{\begin{array}{l}
\left.-\left(p u^{\prime}\right)^{\prime}+q u=\lambda f(t, u) \quad \text { in } I=\right] a, b[ \\
u(a)=u^{\prime}(b)=0
\end{array}\right.
$$

where $p, q \in L^{\infty}([a, b])$ are such that

$$
p_{0}=\underset{t \in[a, b]}{\operatorname{essinf}} p(t)>0, \quad q_{0}=\underset{t \in[a, b]}{\operatorname{essinf}} q(t) \geq 0,
$$

$f:[a, b] \times \mathbb{R} \rightarrow \mathbb{R}$ is a Carathéodory function and $\lambda$ is a positive real parameter.

Put

$$
F(t, x)=\int_{0}^{x} f(t, \xi) d \xi
$$

for all $(t, x) \in[a, b] \times \mathbb{R}$. Denote

$$
X=\left\{u \in W^{1,2}([a, b]): u(a)=0\right\} ;
$$

the usual norm in $X$ is defined by

$$
\|u\|_{X}=\left(\int_{a}^{b} u^{2}(t) d t+\int_{a}^{b}\left(u^{\prime}(t)\right)^{2} d t\right)^{1 / 2} .
$$

For every $u, v \in X$, we define

$$
(u, v)=\int_{a}^{b} p(t) u^{\prime}(t) v^{\prime}(t) d t+\int_{a}^{b} q(t) u(t) v(t) d t .
$$

We observe that (2) defines an inner product on $X$ whose corresponding norm is

$$
\|u\|=\left(\int_{a}^{b} p(t)\left(u^{\prime}(t)\right)^{2} d t+\int_{a}^{b} q(t)(u(t))^{2} d t\right)^{1 / 2} .
$$

A simple computation shows that the norm $\|\cdot\|$ is equivalent to the usual one.

A function $u \in X$ is said to be a weak solution of problem $\left(R S_{\lambda}\right)$ if

$$
\int_{a}^{b} p(t) u^{\prime}(t) v^{\prime}(t) d t+\int_{a}^{b} q(t) u(t) v(t) d t=\lambda \int_{a}^{b} f(t, u(t)) v(t) d t \quad \forall v \in X .
$$

Clearly, if $f$ is continuous, $p \in C^{1}([a, b])$ and $q \in C^{0}([a, b])$, then weak solutions of $\left(R S_{\lambda}\right)$ are classical solutions. and

It is well known that $(X,\|\cdot\|)$ is compactly embedded in $\left(C^{0}([a, b]),\|\cdot\|_{\infty}\right)$

$$
\|u\|_{\infty} \leq \sqrt{\frac{b-a}{p_{0}}}\|u\| \quad \forall u \in X
$$


We use the following notations:

$$
\|p\|_{\infty}=\operatorname{esssup}_{t \in[a, b]} p(t), \quad\|q\|_{\infty}=\operatorname{ess~sup}_{t \in[a, b]} q(t) .
$$

In order to study problem $\left(R S_{\lambda}\right)$, we will use the functionals $\Phi, \Psi: X \rightarrow \mathbb{R}$ defined by putting

$$
\Phi(u)=\frac{1}{2}\|u\|^{2}, \quad \Psi(u)=\int_{a}^{b} F(t, u(t)) d t \quad \forall u \in X .
$$

Since $\Phi$ is continuous and convex it is weakly sequentially lower semicontinuous. Moreover $\Phi$ is continuously Gâteaux differentiable and its Gâteaux derivative admits a continuous inverse. On the other hand, $\Psi$ is sequentially weakly upper semicontinuous, Gâteaux differentiable with compact derivative, one has

$$
\begin{aligned}
& \Phi^{\prime}(u)(v)=\int_{a}^{b} p(t) u^{\prime}(t) v^{\prime}(t) d t+\int_{a}^{b} q(t) u(t) v(t) d t, \\
& \Psi^{\prime}(u)(v)=\int_{a}^{b} f(t, u(t)) v(t) d t \quad \forall v \in X,
\end{aligned}
$$

and moreover

$$
\Phi(0)=\Psi(0)=0 .
$$

Since a critical point for the functional $\Phi-\lambda \Psi$ is any $u \in X$ such that

$$
\Phi^{\prime}(u)(v)-\lambda \Psi^{\prime}(u)(v)=0 \quad \forall v \in X,
$$

the critical points for $\Phi-\lambda \Psi$ are exactly the weak solutions for problem $\left(R S_{\lambda}\right)$.

Now, put

$$
\begin{aligned}
k & =\frac{3 p_{0}}{6\|p\|_{\infty}+2(b-a)^{2}\|q\|_{\infty}}, \\
A & =\liminf _{\xi \rightarrow+\infty} \frac{\int_{a}^{b} \max _{|x| \leq \xi} F(t, x) d t}{\xi^{2}}, \\
B & =\limsup _{\xi \rightarrow+\infty} \frac{\int_{(a+b) / 2}^{b} F(t, \xi) d t}{\xi^{2}}, \\
\lambda_{1} & =\frac{1}{3(b-a)} \frac{3\|p\|_{\infty}+(b-a)^{2}\|q\|_{\infty}}{B}=\frac{p_{0}}{2(b-a) k B}, \\
\lambda_{2} & =\frac{p_{0}}{2(b-a) A} .
\end{aligned}
$$

We note that if $p=q=1$ and $b-a=1$ then $k=3 / 8$, and if $q=0$ and $p=1$ then $k=1 / 2$. 
3. Mixed boundary value problems. Our main result is the following theorem.

Theorem 3.1. Assume that

$$
(a+b) / 2
$$

i) $\quad \int_{a} F(t, \xi) d t \geq 0 \quad \forall \xi \geq 0$,

(ii) $\liminf _{\xi \rightarrow+\infty} \frac{\int_{a}^{b} \max _{|x| \leq \xi} F(t, x) d t}{\xi^{2}}<k \limsup _{\xi \rightarrow+\infty} \frac{\int_{(a+b) / 2}^{b} F(t, \xi) d t}{\xi^{2}}$

where $k$ is given by (5).

Then, for each $\lambda \in] \lambda_{1}, \lambda_{2}\left[\right.$, where $\lambda_{1}, \lambda_{2}$ are given by (8) and (9), the problem $R S_{\lambda}$ has a sequence of weak solutions which is unbounded in $X$.

Proof. Our goal is to apply Theorem 2.1. Consider the Sobolev space $X$ and the operators defined in (4). Pick $\lambda \in] \lambda_{1}, \lambda_{2}[$.

Let $\left\{c_{n}\right\}$ be a real sequence such that $\lim _{n \rightarrow+\infty} c_{n}=+\infty$ and

$$
\lim _{n \rightarrow+\infty} \frac{\int_{a}^{b} \max _{|\xi| \leq c_{n}} F(t, \xi) d t}{c_{n}^{2}}=A .
$$

Put

$$
r_{n}=\frac{p_{0}}{2(b-a)} c_{n}^{2} \quad \text { for all } n \in \mathbb{N} .
$$

Taking into account (3), one has $\|v\|_{\infty} \leq c_{n}$ for all $v \in X$ such that $\|v\|^{2} \leq$ $2 r_{n}$. Hence, for all $n \in \mathbb{N}$,

$$
\begin{aligned}
\varphi\left(r_{n}\right) & =\inf _{u \in \Phi^{-1}(]-\infty, r_{n}[)} \frac{\sup _{v \in \Phi^{-1}(]-\infty, r_{n}[)} \Psi(v)-\Psi(u)}{r_{n}-\Phi(u)} \\
& \leq \frac{\sup _{\|v\|^{2}<2 r_{n}} \int_{a}^{b} F(t, v(t)) d t}{r_{n}} \\
& \leq \frac{\int_{a}^{b} \max _{|\xi| \leq c_{n}} F(t, \xi) d t}{r_{n}}=\frac{2(b-a)}{p_{0}} \frac{\int_{a}^{b} \max _{|\xi| \leq c_{n}} F(t, \xi) d t}{c_{n}^{2}} .
\end{aligned}
$$

Therefore, since from (ii) one has $A<\infty$, we obtain

$$
\gamma:=\liminf _{n \rightarrow \infty} \varphi\left(r_{n}\right) \leq \frac{2(b-a)}{p_{0}} A<\infty .
$$

Now, we claim that the functional $I_{\lambda}=\Phi-\lambda \Psi$ is unbounded from below. Let $\left\{d_{n}\right\}$ be a real sequence such that $\lim _{n \rightarrow \infty} d_{n}=+\infty$ and

$$
\lim _{n \rightarrow+\infty} \frac{\int_{(a+b) / 2}^{b} F\left(t, d_{n}\right) d t}{d_{n}^{2}}=B .
$$


For all $n \in \mathbb{N}$ define

$$
\omega_{n}(t)= \begin{cases}\frac{2 d_{n}}{b-a}(t-a) & \text { if } t \in[a,(a+b) / 2[, \\ d_{n} & \text { if } t \in[(a+b) / 2, b] .\end{cases}
$$

Clearly, $\omega_{n} \in X$ and

$$
\left\|\omega_{n}\right\|^{2} \leq 2 \frac{d_{n}^{2}}{b-a}\left(\|p\|_{\infty}+\frac{(b-a)^{2}}{3}\|q\|_{\infty}\right)
$$

Therefore

$$
\begin{aligned}
\Phi\left(\omega_{n}\right)-\lambda \Psi\left(\omega_{n}\right) & =\frac{1}{2}\left\|\omega_{n}\right\|^{2}-\lambda \int_{a}^{b} F\left(t, \omega_{n}(t)\right) d t \\
\leq & \frac{d_{n}^{2}}{b-a}\left(\|p\|_{\infty}+\frac{(b-a)^{2}}{3}\|q\|_{\infty}\right)-\lambda \int_{a}^{b} F\left(t, \omega_{n}(t)\right) d t .
\end{aligned}
$$

Taking into account (i), we have

$$
\int_{a}^{b} F\left(t, \omega_{n}(t)\right) d t \geq \int_{(a+b) / 2}^{b} F\left(t, d_{n}\right) d t .
$$

Then, for all $n \in \mathbb{N}$,

$$
\begin{aligned}
\Phi\left(\omega_{n}\right)-\lambda \Psi\left(\omega_{n}\right) & \leq \frac{d_{n}^{2}}{b-a}\left(\|p\|_{\infty}+\frac{(b-a)^{2}}{3}\|q\|_{\infty}\right)-\lambda \int_{(a+b) / 2}^{b} F\left(t, d_{n}\right) d t \\
& =\frac{d_{n}^{2} p_{0}}{2(b-a) k}-\lambda \int_{(a+b) / 2}^{b} F\left(t, d_{n}\right) d t .
\end{aligned}
$$

Now, if $B<\infty$, we fix $\epsilon \in] \frac{p_{0}}{2 \lambda(b-a) k B}, 1\left[\right.$. From $\sqrt{10}$ there exists $\nu_{\epsilon} \in \mathbb{N}$ such that

$$
\int_{(a+b) / 2}^{b} F\left(t, d_{n}\right) d t>\epsilon B d_{n}^{2} \quad \forall n>\nu_{\epsilon}
$$

therefore

$$
\Phi\left(\omega_{n}\right)-\lambda \Psi\left(\omega_{n}\right) \leq\left[\frac{p_{0}}{2(b-a) k}-\lambda \epsilon B\right] d_{n}^{2} \quad \forall n>\nu_{\epsilon},
$$

and by the choice of $\epsilon$, one has

$$
\lim _{n \rightarrow \infty}\left[\Phi\left(\omega_{n}\right)-\lambda \Psi\left(\omega_{n}\right)\right]=-\infty
$$

On the other hand, if $B=+\infty$, we fix

$$
M>\frac{p_{0}}{2 \lambda(b-a) k} \text {. }
$$


From 10 there exists $\nu_{M} \in \mathbb{N}$ such that

$$
\int_{(a+b) / 2}^{b} F\left(t, d_{n}\right) d t>M d_{n}^{2} \quad \forall n>\nu_{M},
$$

therefore

$$
\Phi\left(\omega_{n}\right)-\lambda \Psi\left(\omega_{n}\right) \leq\left[\frac{p_{0}}{2(b-a) k}-\lambda M\right] d_{n}^{2} \quad \forall n>\nu_{M},
$$

and by the choice of $M$, one has

$$
\lim _{n \rightarrow \infty}\left[\Phi\left(\omega_{n}\right)-\lambda \Psi\left(\omega_{n}\right)\right]=-\infty .
$$

Hence, our claim is proved.

Since all assumptions of Theorem 2.1 are satisfied, the functional $I_{\lambda}=$ $\Phi-\lambda \Psi$ admits a sequence $\left\{u_{n}\right\}$ of critical points such that $\lim _{n \rightarrow \infty}\left\|u_{n}\right\|=$ $+\infty$, and the conclusion is achieved.

Now, we point out the following consequence of Theorem 3.1.

Corollary 3.1. Let $f: \mathbb{R} \rightarrow \mathbb{R}$ be a nonnegative continuous function, $q \in C^{0}([a, b]), p \in C^{1}([a, b])$ and put $F(t)=\int_{0}^{t} f(\xi) d \xi$ for all $t \in \mathbb{R}$. Assume that

$$
\liminf _{\xi \rightarrow+\infty} \frac{F(\xi)}{\xi^{2}}<\frac{k}{2} \limsup _{\xi \rightarrow+\infty} \frac{F(\xi)}{\xi^{2}} .
$$

Then, for each $\lambda$ in the interval

$$
] \frac{p_{0}}{(b-a)^{2} k \lim \sup _{\xi \rightarrow+\infty} F(\xi) / \xi^{2}}, \frac{p_{0}}{2(b-a)^{2} \liminf _{\xi \rightarrow+\infty} F(\xi) / \xi^{2}}[,
$$

the problem

$$
\left\{\begin{array}{l}
\left.-\left(p u^{\prime}\right)^{\prime}+q u=\lambda f(u) \quad \text { in } I=\right] a, b[ \\
u(a)=u^{\prime}(b)=0
\end{array}\right.
$$

possesses a sequence of pairwise distinct classical solutions.

Remark 3.1. In Theorem 3.1 we can replace $\xi \rightarrow+\infty$ by $\xi \rightarrow 0^{+}$, applying in the proof part (c) of Theorem 2.1 instead of (b). In this case we obtain a sequence of pairwise distinct solutions to the problem $R S_{\lambda}$ which converges uniformly to zero.

Now, consider the following problem:

$$
\left\{\begin{array}{l}
\left.-\left(\bar{p} u^{\prime}\right)^{\prime}+\bar{r} u^{\prime}+\bar{q} u=\lambda g(t, u) \quad \text { in } I=\right] a, b[ \\
u(a)=u^{\prime}(b)=0
\end{array}\right.
$$

where $g:[a, b] \times \mathbb{R} \rightarrow \mathbb{R}$ is a continuous function, $\bar{p} \in C^{1}([a, b]), \bar{q}, \bar{r} \in$ $C^{0}([a, b])$ and $\lambda$ is a positive parameter. Moreover, $\bar{p}$ is positive, $\bar{q}$ is nonnegative and $R$ is a primitive of $\bar{r} / \bar{p}$. 
Put

$$
G(t, x)=\int_{0}^{x} g(t, \xi) d \xi
$$

for all $(t, x) \in[a, b] \times \mathbb{R}$, and

$$
\begin{aligned}
k^{\prime} & =\frac{3 \min _{t \in[a, b]} e^{-R} \bar{p}}{6\left\|e^{-R} \bar{p}\right\|_{\infty}+2(b-a)^{2}\left\|e^{-R} \bar{q}\right\|_{\infty}}, \\
\bar{\lambda}_{1} & =\frac{1}{3(b-a)} \frac{3\left\|e^{-R} \bar{p}\right\|_{\infty}+(b-a)^{2}\left\|e^{-R} \bar{q}\right\|_{\infty}}{\limsup _{\xi \rightarrow+\infty} \int_{(a+b) / 2}^{b} e^{-R(t)} G(t, \xi) d t / \xi^{2}}, \\
\bar{\lambda}_{2} & =\frac{\min _{t \in[a, b]} e^{-R} \bar{p}}{2(b-a) \liminf _{\xi \rightarrow+\infty} \int_{a}^{b} \max _{|x| \leq \xi} e^{-R(t)} G(t, x) d t / \xi^{2}} .
\end{aligned}
$$

Corollary 3.2. Assume that

$$
\int_{a}^{(a+b) / 2} e^{-R(t)} G(t, \xi) d t \geq 0 \quad \forall \xi \geq 0
$$

(i)

(ii) $\liminf _{\xi \rightarrow+\infty} \frac{\int_{a}^{b} \max _{|x| \leq \xi} e^{-R(t)} G(t, x) d t}{\xi^{2}}<k^{\prime} \limsup _{\xi \rightarrow+\infty} \frac{\int_{(a+b) / 2}^{b} e^{-R(t)} G(t, \xi) d t}{\xi^{2}}$.

Then, for each $\lambda \in] \bar{\lambda}_{1}, \bar{\lambda}_{2}\left[\right.$, the problem $\left(P_{\lambda}\right)$ possesses a sequence of pairwise distinct classical solutions.

Proof. Since the solutions of the problem

$$
\left\{\begin{array}{l}
\left.-\left(e^{-R} \bar{p} u^{\prime}\right)^{\prime}+e^{-R} \bar{q} u=\lambda e^{-R} g(t, u) \quad \text { in } I=\right] a, b[ \\
u(a)=u^{\prime}(b)=0
\end{array}\right.
$$

are solutions of the problem $\left(P_{\lambda}\right)$, the conclusion follows from Theorem 3.1.

REMARK 3.2. Theorem 1.1 in the Introduction immediately follows from Corollary 3.2 .

ExAmPle 3.1. Put

$$
a_{n}:=\frac{2 n !(n+2) !-1}{4(n+1) !}, \quad b_{n}:=\frac{2 n !(n+2) !+1}{4(n+1) !}
$$

for every $n \in \mathbb{N}$, and define the nonnegative continuous function $g: \mathbb{R} \rightarrow \mathbb{R}$ by

$$
g(\xi)=\left\{\begin{array}{rr}
\frac{32(n+1) !^{2}\left[(n+1) !^{2}-n !^{2}\right]}{\pi} \sqrt{\frac{1}{16(n+1) !^{2}}-\left(\xi-\frac{n !(n+2)}{2}\right)^{2}} \\
\text { if } \xi \in \bigcup_{n \in \mathbb{N}}\left[a_{n}, b_{n}\right] \\
\text { otherwise. }
\end{array}\right.
$$


By a simple computation, we obtain

so

$$
\liminf _{\xi \rightarrow+\infty} \frac{G(\xi)}{\xi^{2}}=0 \quad \text { and } \quad \limsup _{\xi \rightarrow+\infty} \frac{G(\xi)}{\xi^{2}}=4
$$

$$
0=\liminf _{\xi \rightarrow+\infty} \frac{G(\xi)}{\xi^{2}}<\frac{3}{2 e(\sqrt{e}+1))}=\frac{3}{8 e(\sqrt{e}+1))} \limsup _{\xi \rightarrow+\infty} \frac{G(\xi)}{\xi^{2}} .
$$

Hence, from Corollary 3.2, for each $\lambda>1 / 3$, the problem

$$
\left\{\begin{array}{l}
\left.-u^{\prime \prime}+u^{\prime}+u=\lambda g(u) \text { in }\right] 0,1[ \\
u(0)=u^{\prime}(1)=0
\end{array}\right.
$$

has a sequence of pairwise distinct classical solutions.

\section{References}

[1] B. Ahmad, J. J. Nieto and N. Shahzad, Generalized quasilinearization method for mixed boundary value problems, Appl. Math. Comput. 133 (2002), 423-429.

[2] D. Averna and R. Salvati, Three solutions for a mixed boundary value problem involving the one-dimensional p-laplacian, J. Math. Anal. Appl. 298 (2004), 245-260.

[3] G. Bonanno and G. Molica Bisci, Infinitely many solutions for a boundary value problem with discontinuous nonlinearities, Boundary Value Probl. 2009, art. ID 670675, $20 \mathrm{pp}$.

[4] D. Kong, L. Liu and Y. Wu, Triple positive solutions of a boundary value problem for nonlinear singular second-order differential equations of mixed type with p-laplacian, Comput. Math. Appl. 58 (2009), 1425-1432.

[5] I. Rachůnková, Singular mixed boundary value problem, J. Math. Anal. Appl. 320 (2006), 611-618.

[6] B. Ricceri, A general variational principle and some of its applications, J. Comput. Appl. Math. 113 (2000), 401-410.

[7] R. Salvati, Multiple solutions for a mixed boundary value problem, Math. Sci. Res. J. 7 (2003), 275-983.

[8] D. Zhao, H. Wang and W. Ge, Existence of triple positive solutions to a class of p-laplacian boundary value problems, J. Math. Anal. Appl. 328 (2007), 972-983.

Gabriele Bonanno

Department of Science

for Engineering and Architecture

(Mathematics Section)

Engineering Faculty

University of Messina

98166 Messina, Italy

E-mail: bonanno@unime.it
Elisabetta Tornatore

Dipartimento di Metodi e Modelli Matematici

Facoltà di Ingegneria Università degli Studi di Palermo Viale delle Scienze

90100 Palermo, Italy E-mail: elisa@math.unipa.it

Received 5.12.2009

and in final form 1.2.2010 
